\title{
Vascular Endothelial Growth Factor (VEGF) Expression in Oral Cancer and its Correlation with Clinico-Pathological Parameters- A Prospective Study
}

\author{
Ashish Singhal ${ }^{1}$, Rahat Hadi ${ }^{2}$, Charu Mahajan ${ }^{3}$, Nuzhat Husain ${ }^{4}$ \\ ${ }^{1}$ Professor and HOD, Department of Surgical Oncology, Dr. Ram Manohar Lohia Institute of Medical Sciences, \\ Lucknow, Uttar Pradesh, India. \\ ${ }^{2}$ Professor, Department of Radiation Oncology, Dr. Ram Manohar Lohia Institute of Medical Sciences, \\ Lucknow, Uttar Pradesh, India. \\ ${ }^{3}$ Ex-Senior Resident, Department of Surgical Oncology, Dr. Ram Manohar Lohia Institute of Medical Sciences, \\ Lucknow, Uttar Pradesh, India. \\ ${ }^{4}$ Professor and HOD, Department of Pathology, Dr. Ram Manohar Lohia Institute of Medical Sciences, \\ Lucknow, Uttar Pradesh, India.
}

\section{ABSTRACT}

\section{BACKGROUND}

Angiogenesis is essential for solid tumour growth and metastasis. VEGF is considered as a surrogate marker of angiogenesis and is a key mediator of hematogenous and lymphatic spread. VEGF may also have a correlation with clinico-pathological parameters. We wanted to study the relation of VEGF expression in oral cancer with clinico-pathological parameters.

\section{METHODS}

This is a cross sectional study of 40 patients of oral cancer conducted at a tertiary care cancer center. The patients underwent complete clinical and radiological assessment along with biopsy before starting treatment. The clinico-pathological parameters of the patients were recorded. VEGF expression was evaluated in the biopsy tissues using immunohistochemistry (IHC) and VEGF score was calculated. It is then correlated with clinico-pathological parameters like age, site of tumour, addictive habits, nodal status, mandibular invasion, stage and grade of the tumour.

\section{RESULTS}

It is found that VEGF expression was not related to the site of tumour, age and addictive habits of the patients. VEGF expression increased with the stage of the tumour. Patients with mandible involvement and nodal involvement had significantly higher VEGF expression with $p$ value of 0.035 and 0.0001 respectively. VEGF expression was less in well differentiated as compared to poorly differentiated tumours $(\mathrm{p}=0.043)$.

\section{CONCLUSIONS}

VEGF expression increased with stage, nodal involvement and the grade of tumour.
Corresponding Author: Rahat Hadi,

Professor,

Department of Radiation Oncology,

Dr. RMLIMS, Vibhuti Khand,

Lucknow-226010, U. P., India.

E-mail: drrahathadi@yahoo.co.in

DOI: $10.14260 /$ jemds/2019/537

Financial or Other Competing Interests: None.

How to Cite This Article:

Singhal A, Hadi R, Mahajan C, et al. Vascular endothelial growth factor (VEGF) expression in oral cancer and its correlation with clinico-pathological parameters- a prospective study. J. Evolution Med. Dent. Sci. 2019;8(31):2462-2466, DOI: $10.14260 /$ jemds/2019/537

Submission 11-06-2019, Peer Review 15-07-2019, Acceptance 22-07-2019, Published 05-08-2019.

\section{KEY WORDS}

VEGF, Oral Cancer, Immunohistochemistry, Grade 


\section{BACKGROUND}

Oral cancer is the sixth most common cancer worldwide with an estimated incidence of 700,000 cases reported annually. ${ }^{1}$ The incidence is highest in India, especially among males. Use of tobacco in any form is a major risk factor for squamous cell cancer (SCC) and it acts synergistically with alcohol in oral cancer pathogenesis. Exposure to these agents results in alteration of genes that are important in the regulation of various cellular functions. Some of the changes include acquisition of immortality, ability to invade tissue and / or metastasize to other sites, as well as the acquiring the ability to induce angiogenesis. ${ }^{2}$ Angiogenesis is essential for solid tumour growth and it facilitates tumour progression and metastasis. Some of these angiogenic factors act directly as mitogenic factors on endothelial cells viz. basic fibroblast growth factor, TGF (Tumour Growth Factor) - $\beta$ and VEGF. VEGF is considered the most prominent of all these factors and is recognized as the key mediator of angiogenesis in different types of cancers. ${ }^{3}$ VEGF is a disulfide linked dimeric glycoprotein and its gene is located on chromosome 6 (6p12). VEGF increases blood vessel permeability, endothelial cell growth, proliferation, migration and differentiation ${ }^{4}$. VEGF family currently includes six members: VEGF-A, Placenta growth factor, VEGF- B, VEGF-C, VEGF-D and orf virus (called VEGF-E). ${ }^{5}$ The founding member, VEGF-A plays an essential role in angiogenesis and closely related to micro vessel density. The major function of VEGF-C appears to be regulation of lymphatic vessel growth and mediate spread of cancer cells through lymphatic channels. VEGF-D also acts on the same receptors as VEGF-C. VEGF $\mathrm{C}$ and $\mathrm{D}$ are not related to microvessel density but incidence of lymph node involvement is higher in VEGF-C and D tumours than negative tumours. ${ }^{5}$ Thus, tumour invasion and lymph node metastasis is closely related to VEGF C and D expression. VEGF A and B expression was detected in both node positive and node negative SCC. The degree of micro-vascularisation may have implication on pathology and treatment. Tumours with higher degrees of vascularisation have easy access to the circulation for metastasis and provide less hypoxic environment for tumour cells rendering them more susceptible to radiation. Thus, neoangiogenesis appears to coincide with the development of tumour metastasis and bears adverse prognostic significance. ${ }^{6}$

\section{Aim of the Study}

We investigated the VEGF expression in biopsy specimens and tried to relate it with clinical TNM (tumour, node, and metastasis) staging, morphology of the tumour, pathological TNM, lymphovascular invasion, extracapsular extension and grade of the tumour.

\section{METHODS}

Patients presenting with early and locally advanced oral cancer having good performance status undergoing treatment were included in the cross-sectional study after taking proper voluntary consent. VEGF expression of the cancerous tissue was studied in all the patients before definitive treatment. The VEGF score was correlated with clinico-pathological parameters. In patients with oral cancer clinical parameters i.e. age, sex, clinical examination findings were noted. The performance status was assessed. The staging work up was done for loco-regional extent of disease (OPG, orthopantomogram/CT, computerized tomography/MRI magnetic resonance imaging). Punch biopsy was taken from the tumour and histological analysis and VEGF status was done on the tumour sample.

\section{Laboratory Technique}

Tissues were obtained using punch biopsy under local anaesthesia before treatment. IHC was used to determine the VEGF expression in the tissue. The sections were immunostained with rabbit polyclonal antibody to VEGF (Biogenics laboratories, USA). This antibody is currently available for in vitro diagnostic use and designed for the specific localization of VEGF in formalin fixed paraffin embedded tissue sections. Antigen retrieval was done using citrate buffer for 90 minutes to optimize staining. Blocking was done with $3 \%$ hydrogen peroxide in methanol for 30 minutes. Detection was done using polymer-based kit with horse reddish peroxidase complex and 3- Di-amino benzidine tetra hydrochloride (DAB) as substrate. Brown stained cells indicated positivity. Percent cell expression was done manually, and percent filled area expression was done by morphometry. Five hundred squamous cells were counted in all cases.

The intensity of VEGF staining in 500 squamous cells of each case was calculated as below-

- No. of cells with negative VEGF expression x $0=\mathrm{A}$

- No. of cells with $1(+)$ intensity x $1=\mathrm{B}$

- No. of cells with $2(++)$ intensity $\times 2=\mathrm{C}$

- No. of cells with $3(+++)$ intensity $\times 3=\mathrm{D}$

VEGF score is the sum of the above. The minimum and maximum possible limits of the VEGF score using the above method of scoring was 0-1500. VEGF grading for all tumour tissues was done using the total VEGF score which is as follows-

- VEGF Grade VEGF SCORE (Figure 1-4)

- Grade 0 0-50

- Grade 151-500

- Grade 2 501-1000

- Grade 3 1001-1500

Tissues with greater than $50 \%$ positive cells were considered to have high VEGF expression. Details of clinicpathological findings were recorded on the standard format.

\section{Statistical Analysis}

The statistical analysis was done using SPSS (Statistical package for social sciences) version 15.0 statistical analysis software. The values were represented in number (\%) and mean +- SD (Standard deviation). Following statistical tests were used. Analysis of variance (ANOVA): The ANOVA test was used to compare the within group and between group variances amongst the study groups. ANOVA provided F ratio, where a higher $F$ value depicted a higher inter-group difference. Student $t$ test is used to test the significance of two means the student $t$ test was used. "p" is level of significance. Factor with $\mathrm{p}<0.05$ will be considered statistically significant. 


\section{RESULTS}

A total of 40 patients with SCC of the oral cavity were recruited and met the criteria for inclusion in the study and completed the study protocol. Following are the baseline characteristics of the study subjects $(n=40)$

\section{Age Distribution}

Majority i.e. 25 out of $40(62.5 \%)$ of our patients were in the age range of $40-50$ years. the minimum age was 27 years and the maximum age was 70 years. Six (15\%) patients were below 40 years and $9(22.5 \%)$ patients were above 50 years.

\section{Gender}

Thirty-three (82.5\%) patients were male and 7 (17.5\%) patients were females. Thus, oral cancer is significantly more common in males as compared to females in our country. This may be attributed to the increased consumption of tobacco in males as compared to females.

\section{Risk Factor}

Tobacco chewing in the form of betel quid or gutka was the most common risk factor of oral cancer in our patients. The duration varies from 5- 20 years. Seventeen (42.5\%) patients also had history of cigarette smoking along with tobacco chewing. Eight (20\%) patients had history of alcohol intake also.

\section{Site}

Alveolo-buccal complex was the most common primary tumour site in our study i.e. 26 (65\%) patients, 8 patients $(20$ $\%)$ had primary tongue cancer involving lateral border. One patient (2.5\%) had floor of mouth cancer and 5 (12.5\%) had carcinoma lip. Thus; alveolo-buccal complex is the most common subsite which may be attributed to the habit of retaining betel quid in the gingivo-buccal sulcus. The corresponding VEGF scores for the various subsites were also studied after stage adjustment. No association of VEGF score with the tumour site was found.

\section{Morphology}

Eighteen (45\%) had ulceroproliferative growth. Twenty-one (52. 5\%) patients had ulcerative growth and 1 patient $(2.5 \%)$ had infiltrative growth on clinical examination. The mean VEGF scores of ulceroproliferative, ulcerative and infiltrative morphology were 996.67, 1028.57 and 1250 respectively. Thus, VEGF scores were not comparable in the three groups due to less number of patients in the infiltrative group.

\section{TNM Staging}

Four (10\%) patients in the study had early stage oral cancer whereas 36 (90\%) patients had locally advanced oral cancer (i.e. Stage 3 and 4). The mean VEGF score in early stage oral cancer patients is 225 whereas in locally advanced stage the mean VEGF score is 111 which is statistically significant $(p<0.05)$. Thus, early stage oral cancer had significantly lower VEGF scores as compared to locally advanced tumours.
- $\quad$ T Stage (Pre-Chemotherapy): Majority of patients i.e. 27 (67.5\%) were of T4 stage. Three $(7.5 \%)$ patients had $\mathrm{T} 3$ tumour, 8 ( 20\% ) patients had T2 tumours and 2 ( $5 \%$ ) patients had T1 tumours. Mean VEGF scores for T1, T2, T3 and T4 tumours were 207.5, 922.5, 950 and 1116.48 respectively. Thus, there was an increasing trend seen in VEGF scores with increasing $\mathrm{T}$ stage which was also statistically significant with a P value of 0.001(table 1).

- $\quad$ N Stage (Pre-Chemotherapy): Mean VEFG scores for node negative and node positive patients were 542.86 and 1120.91 respectively which is statistically significant. Thus, VEGF scores increases significantly with nodal involvement i.e. stage progression with a P value $<0.001$.

\section{Grade of Tumour/Differentiation}

Thirty-one (77.5\%) patients had well differentiated (WD) tumours and $8(20 \%)$ patients had moderately differentiation (MD) tumours. Only one patient had poorly differentiated tumours. The mean VEGF scores for the three groups were 948.87, 1253.3 and 1350 respectively which is statistically significant $(\mathrm{P}=0.043)$. Thus, $\mathrm{VEGF}$ score is inversely related to the degree of differentiation of the tumour (table 1).

\section{Bone Destruction}

Sixteen (14\%) patients had mandibular cortical bone destruction at presentation while $24(60 \%)$ patients had normal mandible (table 1). The mean VEGF scores in patients with mandibular destruction, is significantly higher compared to patients without bone destruction. This may be attributed to higher stage of the tumour in these patients $(\mathrm{P}=0.035)$.

\section{VEGF Score}

All the 40 patients were VEGF positive and had a score ranging from $60-1425$ with a mean of 1023 . Four $(10 \%)$ patients had Grade 1 VEGF positivity (Score 50 - 500), 10 (25\%) patients had Grade 2 VEGF positivity (501 - 1000) and 26 (65\%) patients had Grade 3 VEGF positivity (More than 1000). Thus, Grade 3 VEGF positivity was most common in the cancerous mucosa compared with the surroundings normal mucosa taken as control (Mean VEGF score $=30$ ) (Table 1 ).

\begin{tabular}{|c|c|c|c|c|c|}
\hline & & $\begin{array}{c}\text { Mean VEGF } \\
\text { Score +/- 2 SD }\end{array}$ & \begin{tabular}{|c|} 
No. of \\
Patients
\end{tabular} & Percentage & $\begin{array}{c}p \\
\text { Value }\end{array}$ \\
\hline \multirow{2}{*}{ I- } & Stage I \& II & $225+/-174.12$ & 4 & 10 & \multirow[b]{2}{*}{$<0.0$} \\
\hline & Stage III\& IV & $1108.06+/-211.78$ & 36 & 90 & \\
\hline \multirow{2}{*}{ II } & No nodal involvement & $542.85+/-433.35$ & 7 & 17.5 & \multirow{2}{*}{0.001} \\
\hline & Nodal involvement & $1120.91+/-209.83$ & 33 & 82.5 & \\
\hline \multirow{4}{*}{ III } & T1 & $207.5+-152.03$ & 2 & 5 & \multirow{4}{*}{0.001} \\
\hline & T2 & $922.5+-492.96$ & 8 & 20 & \\
\hline & T3 & $950+-278.39$ & 3 & 7.5 & \\
\hline & $\mathrm{T} 4$ & $1116.48+-188.77$ & 27 & 67.5 & \\
\hline \multirow{3}{*}{ IV } & Well diff & $948.87+-344.99$ & 31 & 77.5 & \multirow{3}{*}{0.043} \\
\hline & Mod diff & $1253.13+-567.71$ & 8 & 20 & \\
\hline & Poorly diff & 1350 & 1 & 2.5 & \\
\hline \multirow{2}{*}{ V } & Bone involvement & $1150.9+-157.75$ & 16 & 40 & \multirow{2}{*}{0.035} \\
\hline & No bone involvement & $928.33+-395.26$ & 24 & 60 & \\
\hline
\end{tabular}



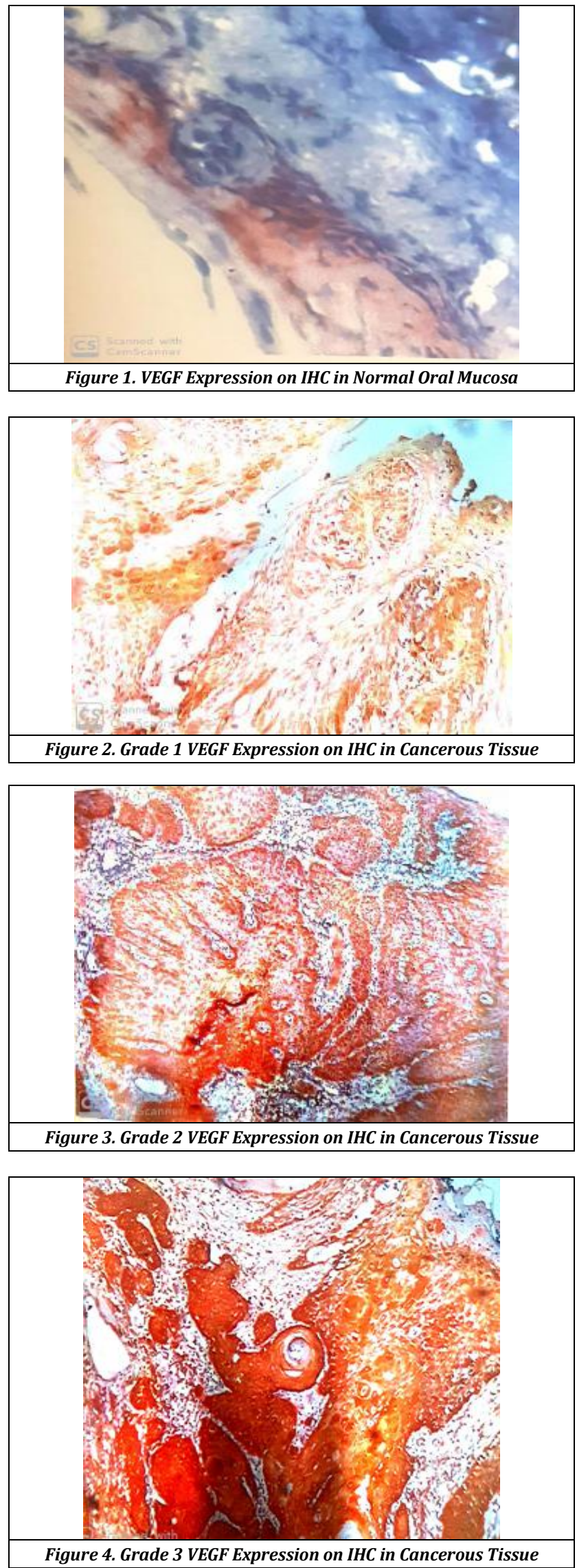

\section{DISCUSSION}

VEGF family members are specific, highly potent angiogenic agents that act to play a major regulatory role in both physiological and pathological angiogenesis as well as lymphangiogenesis. VEGF expression was detected in a relatively low percentage of examined tumours but was found to be associated with the stage of the disease. In contrast, survival analysis failed to establish a prognostic significance of the marker. ${ }^{2}$ The present study is to evaluate VEGF expression in oral cancer and to study the relationship between its expression and clinico-pathological parameters. The majority of patients were above 40 years of age ${ }^{7}(85 \%)$ with a male to female ratio of $4.5: 1$. Most of the patients present with ulcerative lesion in the alveolo-buccal complex followed by tongue and floor of mouth. Majority of patients had locally advanced tumours at presentation. Pandey $\mathrm{M}$ et al, ${ }^{8}$ found that only $2.8 \%$ patients with oral cancer were below 40 years with the male to female ratio of $4.3: 2$. Several studies reported that carcinoma of buccal mucosa constitutes $40-50 \%$ of all cancers of the oral cavity. ${ }^{8,9}$ Chewing of betel quid with tobacco is the main risk factor. In the present study, VEGF was over expressed in malignant tissue as compared to adjacent normal mucosa. Faratzis G et al, ${ }^{2}$ also found that VEGF is over expressed in cancerous mucosa.

In the present study, $90 \%$ of patients presented with advanced oral cancer. This may be due to the fact that patients generally came to our institution after a long period had elapsed between the development of lesion and presentation. VEGF expression was found to increase significantly with advancing stage of tumour. Oral cavity cancers generally present in advanced stage (Stage $3 \& 4$ ) in $60-75 \%$ of cases. Faratzis et $\mathrm{al}^{2}{ }^{2}$ showed that VEGF overexpression was associated with aggressive phenotype and advanced stage of tumour. Yu Hong Li et al,10 found that VEGF expression was higher in stage 3 and 4 tumours as compared to stage $1 \& 2$. However, Shang ZJ et $\mathrm{al}^{11}$ found that VEGF significantly increases in patients with stage $1 \& 2$ tumours as compared to when there are stage $3 \& 4$ tumours. ${ }^{14,15}$ Thus, correlation of VEGF expression with clinical stage is controversial and no definite conclusion can be drawn from such a small sample size.

In the present study, the incidence of nodal positive cases was $82.5 \%$. This may be attributed to higher incidence of infective lymphadenopathy due to poor oral hygiene in these patients. The clinical staging however included these patients as node positive. VEGF expression was significantly higher in patients with regional lymph node involvement. T. Maeda et $\mathrm{al}^{12}$ and B D Smith et al ${ }^{13}$ showed that VEGF expression was significantly higher in patients with regional lymph node involvement. However, Shintani et $\mathrm{al}^{6}{ }^{6}$ found no significant difference between VEGF expression and lymph node involvement.

In the present study, majority of cases was WD followed by MD tumours. This compares favourably with studies conducted by Rajender $\mathrm{R}$ et al and Sherin et $\mathrm{al}^{7}$. VEGF expression was significantly higher in PD and MD tumours as compared to WD tumours. Kyzas PA et al, ${ }^{14}$ found a trend towards modest correlation of VEGF positivity with poor differentiation although the results were not statistically significant. Majority of studies, ${ }^{11,12,13}$ found no significant association between differentiation level of the tumour and VEGF expression. One study conducted by Shintani S et al, 6 however did find a significantly higher number of VEGF positive tumours which were WD compared to moderately or PD tumours. Since, the numbers of patients included in all 
these studies were less including present study; no definite conclusion could be made.

\section{CONCLUSIONS}

A total of 40 patients with early and locally advanced oral cancer were studied. Pre-treatment biopsy was done for tissue diagnosis. The VEGF status was evaluated in biopsy specimen. VEGF was compared between the malignant area and surrounding normal epithelium and was correlated with histopathological parameters. Majority of patients had ulcerative type of lesion that was WD on biopsy. VEGF was significantly less in WD as compared to PD tumours $(\mathrm{p}=0.0430)$. Bone involvement was seen in $60 \%$ of patients due to advanced stage at presentation and proximity of the alveolo-buccal complex to mandible. Patients with mandibular involvement has a significantly higher VEGF expression ( $\mathrm{p}=$ 0.035). Palpable lymphadenopathy was present in $80 \%$ of patients, which was confirmed pathologically in $40 \%$ of cases. VEGF was significantly over-expressed in patients with nodal disease $(p=0.001)$. There was a significant difference in VEGF expression between patients with early stage disease compared to locally advanced disease ( $p<0.001$ ). VEGF expression was not related to the site of tumour, age and addictive habits of the patients. We can therefore conclude from this small study that VEGF expression increases with higher stage and nodal involvement and is inversely related to tumour differentiation.

\section{REFERENCES}

[1] Coelho KR. Challenges of the oral cancer burden in India. Article ID 701932, J Cancer Epidemiol 2012;2012:1-17. doi:10.1155/2012/701932

[2] Faratzis G, Tsiambas E, Rapdis AD, et al. VEGF and KI 67 expression in squamous cell carcinoma of tongue - an immunohistochemical and computerized image analysis study. Oral Oncol 2009;45(7):584-8.
[3] Ellis LM, Rosen L, Gordon MS. Overview of anti-VEGF therapy and angiogenesis. Part 1: angiogenesis inhibition in solid tumour malignancies. Clin Adv Hematol Oncol 2006;4(1):1-10.

[4] Senger DR, Van de Water L, Brown LF, et al. Vascular permeability factor (VPF, VEGF) in tumour biology. Cancer Metastasis Rev 1993;12(3-4):303-24.

[5] Shintani S, Li C, Ishikawa T, et al. Expression of vascular endothelial growth factor $\mathrm{A}, \mathrm{B}, \mathrm{C}$ and $\mathrm{D}$ in oral squamous cell carcinoma. Oral Oncol 2004;40(1):1320.

[6] Folkman J, Shing Y. Angiogenesis. J Biol Chem 1992;267(16):10931-4.

[7] Sherin N, Simi T, Shameena P, et al. Changing trend in oral cancer. Indian J of Cancer 2008;45(3):93-6.

[8] Iype EM, Pandey M, Mathew A, et al. Oral cancer among patients under the age of 35 years. Journal of Postgraduate Medicine 2001;47(3):171-6.

[9] Dinshaw KA, Rao DN. Hospital cancer registry annual report 1998, Mumbai: TMH 2002: p. 23-9.

[10] $\mathrm{Li} \mathrm{YH,} \mathrm{Hu} \mathrm{CF,} \mathrm{Shao} \mathrm{Q} \mathrm{et} \mathrm{al.} \mathrm{Elevated} \mathrm{expression} \mathrm{of}$ survival and VEGF protein are strong independent predictors of survival in advanced nasopharyngeal carcinoma. Journal of Translational Medicine 2008;6:1.

[11] Shang ZJ, Li JR. Expression of endothelial nitric oxide synthase and vascular endothelial growth factor in oral squamous cell carcinoma its correlation with angiogenesis and disease progression. J Oral Pathol Med 2005;34(3):134-9.

[12] Maeda T, Matsumura S, Hiranuma H, et al. Expression of vascular endothelial growth factors in human oral squamous cell carcinoma its association with tumour progression and p53 gene status. J Clin Pathol 1998;51(10):771-5.

[13] Smith BD, Smith GL, Carter D, et al. Prognostic significance of vascular endothelial growth factor level in oral and oropharyngeal squamous cell carcinoma. J Clin Oncol 2000;18(10):2046-52.

[14] Kyzas PA, Cunha IW, Ioannidis JP. Prognostic significance of VEGF immunohistochemical expression in head and neck squamous cell carcinoma - a metaanalysis. Clinical Cancer Research 2005;11(4):1434-40. 